\title{
Efficacy and safety of amrubicin hydrochloride for treatment of relapsed small cell lung cancer
}

This article was published in the following Dove Press journal:

Cancer Management and Research

10 August 2010

Number of times this article has been viewed

\section{Daiki Ogawara' \\ Minoru Fukuda ${ }^{2}$ \\ Yoichi Nakamura ${ }^{3}$ \\ Shigeru Kohno ${ }^{3}$ \\ 'Department of Medicine, Sasebo Central Hospital, ${ }^{2}$ Department of Medicine, Nagasaki Municipal Hospital, ${ }^{3}$ Second Department of Internal Medicine, Nagasaki University School of Medicine, Nagasaki, Japan}

Correspondence: Minoru Fukuda Department of Medicine, Nagasaki Municipal Hospital, 6-39 Shinchi, Nagasaki, 850-8555, Japan

$\mathrm{Tel}+8 \mid 95822325$ I

Fax +8I 958268798

Email mifukuda258@nifty.com
Abstract: Long-term survival is quite uncommon in refractory small cell lung cancer (SCLC) patients, with less than $25 \%$ of patients with limited-stage disease and $1 \%-2 \%$ of patients with extensive-stage disease remaining alive at five years. Recent clinical studies have demonstrated the promising efficacy of amrubicin for patients with relapsed SCLC. This review presents the results of clinical studies showing the efficacy and safety of amrubicin for the treatment of relapsed SCLC. Amrubicin is a synthetic anthracycline agent with a similar structure to doxorubicin, in which the hydroxyl group at position 9 in amrubicin is replaced by an amino group to enhance efficacy. It is converted to an active metabolite, amrubicinol, which is 5-54 times more active than amrubicin. Amrubicin and amrubicinol are inhibitors of DNA topoisomerase II, exerting their cytotoxic effects by stabilizing a topoisomerase II-mediated cleavable complex. The toxicity of amrubicin is similar to that of doxorubicin, although amrubicin shows almost no cardiotoxicity. In the relevant trials, amrubicin was administered intravenously at a dose of $35-40 \mathrm{mg} / \mathrm{m}^{2}$ on days $1-3$ every three weeks. The response rate was $34 \%-52 \%$ and median survival times were 8.1-12.0 months. Common hematologic toxicities included neutropenia, leucopenia, anemia, thrombocytopenia, and febrile neutropenia. Nonhematologic adverse events included Grade 3-4 anorexia, asthenia, hyponatremia, and nausea. The results of the studies which demonstrated the efficacy of monotherapy for relapsed SCLC involved mainly Japanese patients. Therefore, it is necessary to conduct more clinical studies in non-Japanese patients to confirm the efficacy of amrubicin.

Keywords: amrubicin, amrubicinol, small cell lung cancer, relapse

\section{Introduction}

Small cell lung cancer (SCLC) accounts for approximately $15 \%$ of all lung cancer cases, with two-thirds of patients presenting with extensive disease (ED). Without treatment, tumor progression in patients with SCLC is rapid, with a poor prognosis. However, the disease shows a high response rate to chemotherapy and radiotherapy, except in a low percentage of patients. Treatment options for relapsed SCLC patients remain limited. A randomized trial demonstrated that single-agent topotecan was at least as efficacious as the three-drug combination of cyclophosphamide-doxorubicin-vincristine for the treatment of patients with sensitive relapsed cases. ${ }^{1}$ Response rates and median survival times were $24 \%$ and 25.0 weeks for topotecan, and $18 \%$ and 24.7 weeks for cyclophosphamide-doxorubicin-vincristine, respectively. In previously untreated ED-SCLC, amrubicin yielded an extremely high response rate of $79 \%$ and a median survival time of 11 months, which was comparable with the results achieved with 
platinum. ${ }^{2,3}$ Recently, clinical studies have demonstrated the efficacy of amrubicin in patients with relapsed SCLC. This review presents the results of clinical studies showing the efficacy and safety of amrubicin for the treatment of relapsed SCLC.

\section{Structure and characteristics}

Amrubicin hydrochloride is a synthetic anthracycline agent, with a similar structure to doxorubicin, in which the hydroxyl group at position 9 group in amrubicin is replaced by an amino group to enhance efficacy (see Figure 1). Amrubicin is converted to an active metabolite, amrubicinol, which is 5-54 times more active than amrubicin, through reduction of its C-13 ketone group to a hydroxyl group by carbonyl reductase. ${ }^{4}$ Other enzymes metabolizing amrubicin and amrubicinol are nicotinamide adenine dinucleotide phosphate (NADPH)dependent $\mathrm{P} 450$ reductase and $\mathrm{NAD}[\mathrm{P}] \mathrm{H}$-dependent quinone oxidoreductase. Doxorubicin, amrubicin, and amrubicinol are inhibitors of DNA topoisomerase II, exerting their cytotoxic effects by stabilizing a topoisomerase II-mediated cleavable complex. ${ }^{5}$ In addition, they are more or less only one-tenth as potent as doxorubicin in producing DNA intercalation.

\section{Preclinical studies}

Antitumor activity and toxicologic aspects were first reported for amrubicin by Morisada et al. ${ }^{6}$ They evaluated this agent in six murine systems and nine human tumornude mouse systems, and found that the antitumor activity of amrubicin was superior to adriamycin in human tumor xenografts, and almost equal against murine experimental tumors. They also evaluated toxicity in mice after a bolus intravenous injection. The acute toxic signs were body

\section{Amrubicin}

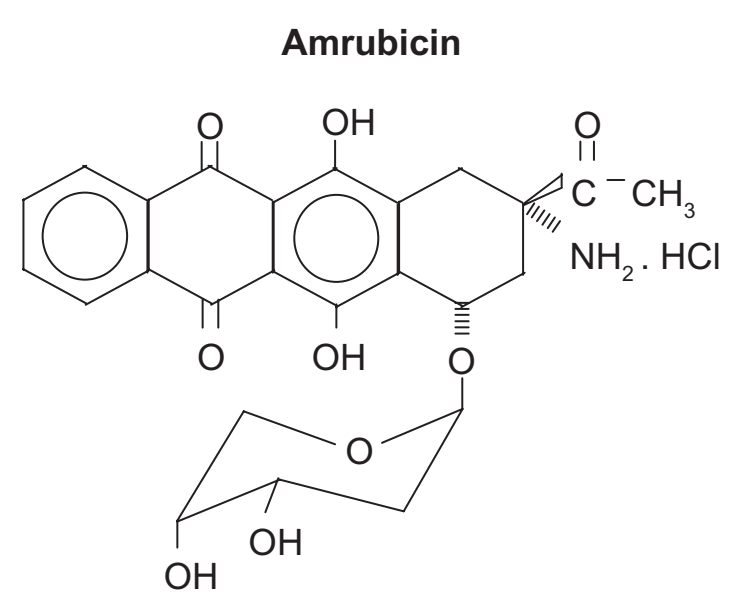

weight decrease, ataxia, hair loss, and myelosuppression, and these toxicities were qualitatively comparable with those induced by adriamycin. The maximum tolerated dose was estimated to be $25 \mathrm{mg} / \mathrm{kg}$ in four mouse strains, and the drug had anticancer activity against human lung cancer xenografts in vivo. ${ }^{\text {? }}$

Cardiomyopathy is a burdensome toxicity with the anthracyclines. Suzuki et al evaluated the degree of cardiotoxicity of amrubicin compared with that of adriamycin in rabbits. ${ }^{8}$ The drugs were intravenously administered three times a week for eight weeks. In this study, prolongation of the QT ${ }_{c}$ interval and STT changes were observed in rabbits administered amrubicin and adriamycin. Morphologic studies showed that myocardial tissue damage in animals administered amrubicin was comparable with that seen in controls. Considering the results of the antitumor efficacy studies comparing amrubicin with adriamycin, they concluded that the cardiotoxicity of amrubicin was very slight.

\section{Relapsed small cell lung cancer}

Long-term survival is quite uncommon in refractory SCLC patients, with less than $25 \%$ of patients with limited-stage disease and $1 \%-2 \%$ of patients with extensive-stage disease remaining alive at five years. A Phase II study was conducted in patients with relapsed disease who had previously received one or two regimens, including at least one regimen of platinum-based chemotherapy ${ }^{9}$ (Table 1). Sixty patients were enrolled in this multicenter trial. The disease progressed within 60 days after the final dose of previous chemotherapy in 16 and 44 refractory patients, respectively. The sensitive groups, in which complete response (CR) or partial response $(\mathrm{PR})$ was observed with previous chemotherapy

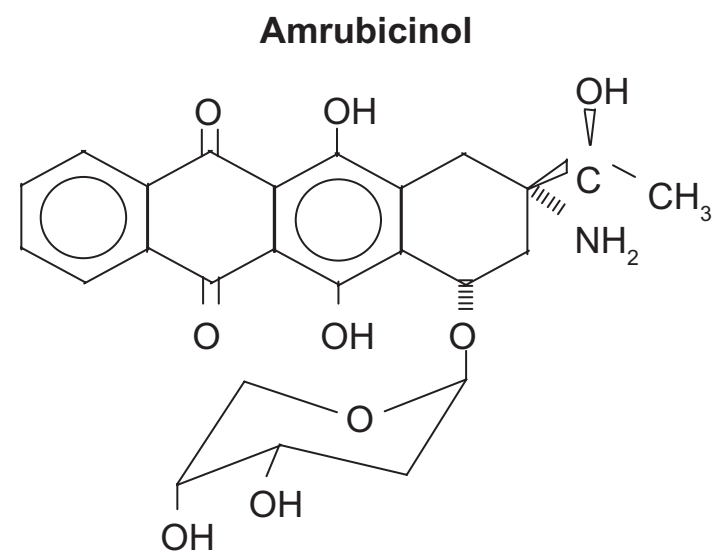

Figure I Chemical structure of amrubicin and amrubicinol. 
Table I Phase II trial of amrubicin for treatment of refractory or relapsed small cell lung cancer (Thoracic Oncology Research Group Study 030I)'

\begin{tabular}{llll}
\hline & Sensitive cases & $\begin{array}{l}\text { Refractory } \\
\text { cases }\end{array}$ & Total \\
\hline Patients $(\mathrm{n})$ & & 16 & 60 \\
CR & 44 & 1 & 2 \\
PR & 1 & 7 & 29 \\
SD & 22 & 2 & 12 \\
PD & 10 & 6 & 17 \\
Response rate $(\%, 95 \% \mathrm{Cl})$ & $\mathrm{II}$ & $50(25 \%-75 \%)$ & $52(38 \%-65 \%)$ \\
PFS (months, 95\% Cl) & $52(37-68)$ & $2.9(1.4-4.6)$ & $3.9(3.4-4.6)$ \\
MST (months, 95\% Cl) & $42(10.0-15.8)$ & $10.3(4.8-\infty)$ & $11.0(10.0-13.2)$ \\
One-year survival $(\%, 95 \% \mathrm{Cl})$ & $11.6(10.0-15.8)$ & $40.3(15.1-64.6)$ & $44.1(30.6-56.8)$ \\
\hline
\end{tabular}

Abbreviations: $\mathrm{Cl}$, confidence interval; $\mathrm{CR}$, complete response; PR, partial response; SD, stable disease; PD, progressive disease; MST, median survival time; PFS, progression-free survival.

and the disease progressed or relapsed within 60 days of the final dose of previous chemotherapy, were eligible for the study, and were assessable for toxicity, response, and survival. Amrubicin was administered intravenously at a dose of $40 \mathrm{mg} / \mathrm{m}^{2}$ on days 1-3 every three weeks. The response rate was 52\% (95\% confidence interval [CI] 38\%-65\%). There were no differences in the response rate, ie, $50 \%$ (95\% CI 25\%-75\%) for refractory disease and 52\% [95\% CI $37 \%-68 \%$ ] for sensitive disease. The median survival times were 10.3 months in the refractory group and 11.6 months in the sensitive group, respectively $(P=0.0974, \log$ rank test). Common adverse events were hematologic toxicities, including Grade 3-4 neutropenia (83\%), leucopenia (70\%), anemia (33\%), thrombocytopenia (20\%), and febrile neutropenia (5\%). Nonhematologic adverse events included Grade 3-4 anorexia (15\%), asthenia (15\%), hyponatremia $(8 \%)$, and nausea $(5 \%)$.

Another Phase II study of amrubicin in patients with previously treated SCLC was conducted by Kaira et a ${ }^{10}$ (Table 2). Twenty-nine patients with relapsed SCLC who had previously received platinum-based chemotherapy were enrolled in the trial, including 10 patients with sensitive relapse and 19 patients with refractory relapse. Amrubicin was administered intravenously at a dose of $35 \mathrm{mg} / \mathrm{m}^{2}$ on

Table 2 Phase II trial of amrubicin for treatment of relapsed small cell lung cancer ${ }^{10}$

\begin{tabular}{llll}
\hline & $\begin{array}{l}\text { Sensitive } \\
\text { cases }\end{array}$ & $\begin{array}{l}\text { Refractory } \\
\text { cases }\end{array}$ & Total \\
\hline Patients (n) & 10 & 19 & 29 \\
$\begin{array}{l}\text { Response rate }(\%, 95 \% \mathrm{Cl}) \\
\begin{array}{l}\text { Progression-free } \\
\text { survival (months) }\end{array}\end{array}$ & 60 & 37 & $45(26-64)$ \\
$\begin{array}{l}\text { Median survival time } \\
\text { (months) }\end{array}$ & 12 & 4 & 4 \\
\hline
\end{tabular}

days $1-3$ every three weeks. The response rate was $44.8 \%$ (95\% CI 26\%-64\%), being 60\% for sensitive cases and $37 \%$ for refractory cases. No significant difference in the response rate was observed between sensitive cases and relapsed cases $(P=0.233, \log$ rank test $)$. The median progression-free survival and median survival times were 4.0 months (sensitive relapse, 4.0 months; refractory relapse, 4.0 months) and 12.0 months (sensitive relapse, 12.0 months; refractory relapse, 11.0 months), respectively. There was no difference in median progression-free survival and median survival time between sensitive relapse and refractory relapse. Grade 3 or 4 neutropenia and febrile neutropenia were observed in $42 \%$ and $3 \%$ of patients, respectively. Nonhematologic toxicity higher than Grade 3 was not observed. The results of this study show the efficacy of monotherapy for relapsed SCLC. However, this study involved only Japanese patients, so it would be necessary to conduct clinical studies in non-Japanese patients to confirm efficacy.

A randomized Phase II trial of amrubicin versus topotecan as second-line treatment for sensitive ED-SCLC was therefore conducted ${ }^{11}$ (Table 3). Seventy-six patients who had previously received platinum-based firstline chemotherapy were enrolled. All were sensitive cases, in which CR or PR had been observed with previous chemotherapy and the disease had then progressed or relapsed within at least 90 days of the final dose. Patients were randomized at a 2:1 ratio to receive either amrubicin or topotecan. Amrubicin was administered intravenously at a dose of $40 \mathrm{mg} / \mathrm{m}^{2}$ on days $1-3$ every three weeks. Topotecan was administered intravenously at a dose of $1.5 \mathrm{mg} / \mathrm{m}^{2}$ on days 1-5 every three weeks. The response rate for amrubicin was $34 \%$ (95\% CI $22 \%-48 \%$ ), and for topotecan was $4 \%$ $(95 \%$ CI $1 \%-19 \%)$. There was a trend towards a longer 
Table 3 Randomized Phase II trial of amrubicin versus topotecan for treatment of sensitive relapsed small cell lung cancer ${ }^{\prime \prime}$

\begin{tabular}{llll}
\hline & Amrubicin & Topotecan & $P$ \\
\hline Patients & 50 & 26 & \\
ORR $(\%, 95 \% \mathrm{Cl})$ & $34(22.4-47.8)$ & $4(0.7-18.9)$ & $<0.004$ \\
CR $(\%, 95 \% \mathrm{Cl})$ & $8(3.2-18.8)$ & 0 & \\
PR $(\%, 95 \% \mathrm{Cl})$ & $26(15.9-39.6)$ & $4(0.7-18.9)$ & \\
$\mathrm{SD}(\%, 95 \% \mathrm{Cl})$ & $32(20.8-45.8)$ & $46(28.8-64.5)$ & \\
PD $(\%, 95 \% \mathrm{Cl})$ & $24(14.3-37.4)$ & $23(11.0-42.1)$ & \\
Median PFS & $138(64-187)$ & $106(75-177)$ & \\
(days, 95\% Cl) & & &
\end{tabular}

Abbreviations: $\mathrm{Cl}$, confidence interval; $\mathrm{CR}$, complete response; $\mathrm{PR}$, partial response; $\mathrm{SD}$, stable disease; $\mathrm{PD}$, progressive disease; PFS, progression-free survival; ORR, overall response rate.

progression-free survival time in the amrubicin group (4.6 months, 95\% CI 64-187) than in the topotecan group (3.5 months, 95\% CI 75-177). This study showed that amrubicin is also active in non-Japanese patients and is well tolerated, with myelotoxicity being the main dose-limiting toxicity. Inoue et al conducted a randomized Phase II trial comparing amrubicin with topotecan in not only sensitive relapsed but also refractory cases $^{12}$ (Table 4). Sensitive cases were defined as CR or PR being achieved with the previous chemotherapy, after which the disease progressed or relapsed at least 90 days after the final dose. Fifty-nine patients were randomized in a 1:1 ratio to receive either amrubicin or topotecan. Amrubicin was administered intravenously at a dose of $40 \mathrm{mg} / \mathrm{m}^{2}$ on days $1-3$ every three weeks. Topotecan was administered intravenously at a dose of $1.0 \mathrm{mg} / \mathrm{m}^{2}$ on days $1-5$ every three weeks. The response rate for amrubicin was 38\% (95\% CI 60\%-92\%) and for topotecan was $13 \%$ (95\% CI 4\%-31\%). In sensitive relapsed cases, the response rate for amrubicin was 53\% (95\% CI $28 \%-77 \%)$, and for topotecan was $21 \%$ (95\% CI $6 \%-46 \%$ ). In refractory relapsed cases, the response rate for

Table 4 Randomized Phase II trial comparing amrubicin with topotecan in patients with sensitive and refractory relapsed small cell lung cancer ${ }^{12}$

\begin{tabular}{|c|c|c|c|}
\hline & Amrubicin & Topotecan & $P$ value \\
\hline Patients (n) & 29 & 30 & \\
\hline ORR (\%, $95 \% \mathrm{Cl})$ & $38(2 \mid-58)$ & $\mid 3(2-3 \mid)$ & 0.039 \\
\hline $\begin{array}{l}\text { ORR in sensitive cases } \\
(\%, 95 \% \mathrm{Cl})\end{array}$ & $53(28-77)$ & $21(6-46)$ & 0.082 \\
\hline $\begin{array}{l}\text { ORR in refractory cases } \\
(\%, 95 \% \mathrm{Cl})\end{array}$ & $67(35-90)$ & $18(2-52)$ & 0.478 \\
\hline Median PFS (months) & 3.5 & 2.2 & 0.16 \\
\hline Overall survival (months) & 8.1 & 8.4 & 0.17 \\
\hline
\end{tabular}

Abbreviations: $\mathrm{Cl}$, confidence interval; ORR, overall response rate; PFS, progression-free survival. amrubicin was $17 \%$ (95\% CI 2\%-48\%), and for topotecan was $0 \%(95 \% \mathrm{CI} 0 \%-28 \%)$. The median progression-free survival time with amrubicin was 3.5 months and with topotecan was 2.2 months. The median overall survival time with amrubicin was 8.1 months and with topotecan was 8.4 months. There was no difference in the frequency of hematologic toxicity more than Grade 3 between amrubicin and topotecan. These studies show that amrubicin monotherapy is an encouraging regimen for second-line treatment of SCLC.

\section{Conclusion}

Clinical investigation of the novel anticancer agent, amrubicin, has increased quickly, and there are high expectations for this agent in trials to improve the outcome for relapsed SCLC patients. Amrubicin is an active agent for the treatment of relapsed SCLC, but because it is strongly myelotoxic, particular care should be taken with its use.

\section{Disclosure}

The authors declare no potential conflicts of interest.

\section{References}

1. von Pawel J, Schiller JH, Shepherd FA, et al. Topotecan versus cyclophosphamide, doxorubicin, and vincristine for the treatment of recurrent small-cell lung cancer. J Clin Oncol. 1999;17: 658-667.

2. Yana T, Negoro S, Takada M, et al. Phase II study of amrubicin in previously untreated patients with extensive-disease small cell lung cancer: West Japan Thoracic Oncology Group (WJOG) study. Invest New Drugs. 2007:25:253-258.

3. Noda K, Nishiwaki Y, Kawahara M, et al. Irinotecan plus cisplatin compared with etoposide plus cisplatin for extensive small-cell lung cancer. N Engl J Med. 2002;346:85-91.

4. Yamaoka T, Hanada M, Ichii S, et al. Cytotoxicity of amrubicin, a novel 9-aminoanthracycline, and its active metabolite amrubicinol on human tumor cells. Jpn J Cancer Res. 1998;89:1067-1073.

5. Hanada M, Mizuno S, Fukushima A, et al. A new antitumor agent amrubicin induces cell growth inhibition by stabilizing topoisomerase II-DNA complex. Jpn J Cancer Res. 1998;89: $1229-1238$.

6. Morisada S, Yanagi Y, Noguchi T, et al. Antitumor activities of novel 9-aminoanthracycline (SM-5887) against mouse experimental tumors and human tumor xenografts. Jpn J Cancer Res. 1989;80: 69-76.

7. Morisada S, Yanagi Y, Kashiwazaki Y, et al. Toxicological aspects of a novel 9-aminoanthracycline, SM-5887. Jpn J Cancer Res. 1989;80:77-82.

8. Suzuki T, Minamide S, Iwasaki T, et al. Cardiotoxicity of a new anthracycline derivative (SM-5887) following intravenous administration to rabbits: Comparative study with doxorubicin. Invest New Drugs. 1997; 15:219-225.

9. Onoda S, Masuda N, Seto T, et al. Phase II trial of amrubicin for treatment of refractory or relapsed small-cell lung cancer. J Clin Oncol. 2006;24:5448-5453. 
10. Kaira K, Sunaga Y, Tomizawa Y, et al. A Phase II study of amrubicin, a synthetic 9-aminoanthracycline, in patients with previously treated lung cancer. Lung Cancer. 2009:3451-3456.

11. Jotte RM, Conkling PR, Reynolds C, et al. A randomized Phase 2 trial of amrubicin (AMR) vs topotecan as second-line treatment in extensivedisease small cell lung cancer (SCLC) sensitive to platinum-based first-line chemotherapy. J Clin Oncol. 2008;26(15S):8040.
12. Inoue A, Sugawara S, Yamazaki K, et al. Randomized phase II trial comparing amrubicin with topotecan in patients with previously treated small-cell lung cancer: North Japan Lung Cancer Study Group Trial 0402. J Clin Oncol. 2008;26:5401-5406.

\section{Publish your work in this journal}

Cancer Management and Research is an international, peer-reviewed open access journal focusing on cancer research and the optimal use of preventative and integrated treatment interventions to achieve improved outcomes, enhanced survival and quality of life for the cancer patient The journal welcomes original research, clinical \& epidemiological studies, reviews \& evaluations, guidelines, expert opinion \& commentary, case reports \& extended reports. The manuscript management system is completely online and includes a very quick and fair peerreview system, which is all easy to use. Visit http://www.dovepress.com/ testimonials.php to read real quotes from published authors.

Submit your manuscript here: http://www.dovepress.com/cancer-management-and-research-journal 